\title{
Association between Children's Appetite Patterns and Maternal Feeding Practices
}

\author{
Tomomi Ainuki, Rie Akamatsu*
}

Graduate School of Humanities and Sciences, Ochanomizu University, Tokyo, Japan.

Email: akamatsu.rie@ocha.ac.jp

Received February $4^{\text {th }}, 2011$; Revised March 10 ${ }^{\text {th }}, 2011$; Accepted Apil $8^{\text {th }}, 2011$.

\begin{abstract}
This study explored associations between children's appetites and maternal feeding practices. The participants, 614 parents of 3- to 6-year-old children from kindergartens in Tokyo, Japan, completed self-administered questionnaires. The children's appetites were measured using the Enjoyment of Food $(E F)$ and Food Responsiveness $(F R)$ scales of the Child Eating Behaviour Questionnaire. Using cluster analysis, we examined three appetite patterns ("both low", "high $E F$ and low FR", and "both high"). The three appetite patterns were associated with children's obesity index. The "both low" pattern was associated with the highest maternal pressure to eat. The "high EF and low FR" patterns were related to an established snack time. The "both high" pattern was associated with higher maternal instrumental feeding compared with the "high EF and low FR" types. The results of this study will facilitate the development of targeted interventions and better parental guidance on maternal feeding practices and their association with children's eating behaviours.
\end{abstract}

Keywords: Appetite, Preschool Children, Feeding Practices

\section{Introduction}

Excessive eating and other issues related to excessive weight have recently emerged as problems in the world [1]. Recent data indicate that $10 \%$ of Japanese children are overweight. However, $10 \%$ of Japanese children are underweight, and it appears that underweight children are prone to undereating [2].

Previous studies have shown that children's appetites are associated with their weight status $[3,4]$. To measure children's appetites, Wardle et al. (2001) developed the Child Eating Behaviour Questionnaire (CEBQ), which included scales assessing such positive appetite traits as Enjoyment of Food (EF) and Food Responsiveness (FR) [5]. Recent studies have compared children's weight status according to each appetite trait on the CEBQ and have found EF and FR to be positively associated with children's weight status [6-8]. Webber et al. (2009) demonstrated that early assessment of appetite traits could be used as an indicator of susceptibility to weight gain [8]. Thus, it is important to examine the research on children's appetites and to study factors associated with children's appetites.

Carnell et al. (2007) argued that both the EF and FR scales address children's general appetite for food or their desire to eat [9]. Higher scores indicate more positive responses to food. Whereas the EF scale aims to capture normal variations in general appetite, the FR items are designed to detect levels of appetite that could be viewed as maladaptive. A previous study examined associations between parental feeding practices and scores on EF and FR [10]. The results showed that high EF was associated with high pressure to eat, and FR was associated with restrictions on eating. In that study, EF and FR were measured and analysed independently. However, in real life, children have both appetite traits, and no study has yet examined the combined effects of EF and FR.

Previous studies have shown that children's appetites were associated with weight status [6-8] and parental feeding practices $[10,11]$. Parents use different feeding practices based on each child's appetite type. Webber et al. (2010) suggested that pressure to eat more healthy foods is a parental response to concerns about a child's not eating enough. That study found that EF was significantly associated with lower maternal pressure to eat, which served to control the child's body mass index [10]. However, FR was not significantly related to maternal 
pressure to eat. Another recent study involving 3- to 6-year-old children showed differences in mothers' use of feeding practices. The results showed that children who scored higher on a measure of responsiveness to external cues or EF were less pressured to eat than were children with lower EF scores [12].

The results of the 2000 Preschool Children's Health Survey in Japan indicate that the proportion of mothers who do not control their children's snacking and do not establish a snack time has increased compared with a decade ago [13]. These environments may contribute to lowering a child's appetite at mealtimes and result in an increased likelihood of snacking; thus, a child's snacking behaviour should also be considered to be related to maternal feeding practices.

The present study examined the characteristics of children's appetite patterns, which were classified based on the CEBQ, and the association between appetite patterns, children's body types, and parental feeding practices. The results will contribute to future intervention related to children's eating behaviours.

\section{Methods}

\subsection{Study Design}

A kindergarten principal who was not involved in this research recruited the six kindergartens in Tokyo that participated in this study. During April and May 2008, packets containing anonymous questionnaires were sent to the principals of each kindergarten. The sample consisted of the parents of children aged 3 to 6 years attending the six kindergartens. The principals distributed the questionnaires to 869 families via the kindergarten teachers. One parent per family completed a self-administered questionnaire focussing on the youngest child in the family who was attending kindergarten; the questionnaire inquired about that child's appetite and the parents' feeding practices with respect to that child. Because fathers and mothers have been shown to feed their children differently [14] and few fathers responded, only data from mothers were included in the analyses. The completed questionnaires were collected at the kindergarten, and the principals gathered them and sent them to the researchers.

The study received approval from the Ochanomizu University Ethical Review Committee.

\subsection{Measures}

\subsubsection{Demographic Characteristics}

The demographic part of the questionnaire included questions about the employment status of the mother, the target child's sex and age, the number of siblings in the family, and the target child's position in the birth order.
Parents also provided information on their child's weight and height, which we used to calculate the obesity index (\{(actual body weight $[\mathrm{kg}]$ - standard body weight)/ standard body weight $\} \times 100$ ). The obesity index is widely used in clinical practices and epidemiological studies in Japan to evaluate children's body types. The standard body weight of infant and preschool children is calculated with a formula based on the Survey on the Growth of Infants and Preschool Children, conducted by the Japan Ministry of Health, Labour and Welfare in 2000 [15]: Boys: $Y=2.06 \times 10^{-3} X^{2}-0.1166 X+6.527$, and Girls: $Y=2.49 \times 10^{-3} X^{2}-0.1858 X+9.0360$, where $Y=$ standard body weight $(\mathrm{kg})$ and $X=$ height $(\mathrm{cm})$. The body index was classified as underweight $(15 \%$ below standard), normal (standard $\pm 15 \%$ ), or overweight (>15\% above standard) as proposed by the Japan Society for the Study of Obesity in 2004 [16].

\subsubsection{Children's Appetites}

Four items measuring EF (e.g., "My child enjoys eating") and five items measuring FR (e.g., "My child is always asking for food") were derived from the Japanese translation of a sub-scale of the CEBQ [5]. Parents responded on a 5-point scale ranging from "never" (1) to "always" (5). The internal reliability values (Chronbach's alpha coefficients) of each EF and FR sub-scale in a previous study were 0.91 and 0.80 , respectively [5].

\subsubsection{Parental Feeding Practices}

Scales were selected from the Child Feeding Questionnaire (CFQ) [17] and the Parental Feeding Style Questionnaire (PFSQ) [18] to address a range of parental feeding behaviours. These questions were translated into Japanese, and their content was confirmed by experts in childcare. Subsequently, five mothers who were not involved in this study reviewed and completed the questionnaires as a pilot study.

The CFQ is composed of 28 questions with seven subscales addressing parental perceptions and behaviours (perceived responsibility, perceived child's weight, concern about child's weight, pressure to eat, food restrictions, and monitoring of eating). Only the sub-scale assessing pressure to eat was used in this study. This 4-item sub-scale asks respondents to use a 5-point scale (ranging from "disagree" to "agree") to rate items addressing their tendency to pressure their children to eat more food (e.g., "I have to be especially careful to make sure my child eats enough"). The internal reliability (Chronbach's alpha coefficient) of this sub-scale in a previous study was 0.70 [17].

The PFSQ consists of 28 questions divided into seven sub-scales that assess parental feeding behaviours (e.g., "emotional feeding", "instrumental feeding", "prompting and providing encouragement to eat", and "control over 
eating"). Only those factors assessing "instrumental feeding" (four items; e.g., "I reward my child with something to eat when she is well-behaved") and "emotional feeding" (five items; e.g., "I give my child something to eat to make him feel better when he is upset") were used in this study. Participants responded on a 5-point scale ranging from "never" (1) to "always" (5). The internal reliability values (Chronbach's alpha coefficient) of the two sub-scales according to a previous study were 0.65 and 0.77 , respectively [18].

\subsubsection{Snacking Behaviour}

Respondents identified the frequency of snacks consumed by their children per day: never, once in a while, once per day, twice per day, and more than three times per day. In addition, they were asked whether they used established snack times.

\subsection{Statistical Analysis}

Demographic information data about respondents and children were analysed. Non-hierarchical cluster analysis was used to identify patterns in the EF and FR scores that were related to children's appetites.

We used the $\chi^{2}$ and Kruskal-Wallis tests to examine the relationships between a child's obesity index, maternal feeding practices, the frequency of snack consumption, the presence of an established snack time, and appetite patterns. For the frequency of snacking, "never" and "once in a while" were combined into "once in a while or less," and "twice a day" and "more than three times a day" were combined into "two or more times a day" in the analysis because the distribution of the respondents was imbalanced. Significance was set at $p<0.05$. The Statistical Package for Social Sciences, SPSS version 18.0, was used for data analysis.

\section{Results}

\subsection{Demographics}

Respondent and child demographic characteristics are shown in Table 1. In total, 614 respondents (70.7\%) completed the survey. The mean age of the children was 4.8 years $(S D: 0.8)$, and the mean number of siblings was 1.9 (SD: 0.7$)$. The majority of respondents were mothers $(99.2 \%)$, most of whom were unemployed.

\subsection{Descriptive Statistics for Children's Appetites and Parental Feeding Practices}

The descriptive statistics for all questions and the data on feeding practices were initially analysed to confirm that the scales had adequate reliability for the present sample. The mean scores were as follows: the mean scores were 3.9 (SD: 0.9) for EF (Chronbach's $\alpha=0.94), 2.2$ (SD: 0.8) for FR $(\alpha=0.84), 3.2(S D: 0.8)$ for pressure to eat $(\alpha=$
$0.64), 2.0(S D: 0.6)$ for instrumental feeding $(\alpha=0.72)$, and $1.5(S D: 0.5)$ for emotional feeding $(\alpha=0.78)$. All scores were consistent with those reported in previous studies $[5,17,18]$.

\subsection{Children's Appetite Patterns}

The three appetite patterns based on the mean EF and FR scores and classified using cluster analysis are shown in Figure 1. The cluster in which EF was lower than average and FR was slightly lower than average was named "both low" ( $n=194)$. Because EF was higher than average and FR was slightly lower than average in the second cluster, that cluster was named "high EF and low FR" (n =290). In the final cluster, named "both high" $(n=118)$, both EF and FR scores were higher than average.

\subsection{Association between Children's Obesity Indices and Appetite Patterns}

The median obesity index for all three appetite patterns was within the normal range, but children's obesity indices differed according to appetite pattern $\left(\chi^{2}=27.8, p<0.001\right)$. Of the three patterns, "both high" was associated with the highest obesity index. The median (25th - 75th percentile)

Table 1. Demographic and anthropometric characteristics of children and families $(n=614)$.

\begin{tabular}{llcc}
\hline & & $n$ & $\%$ \\
\hline \multirow{2}{*}{ Child sex } & Girl & 266 & 43.3 \\
& 1 & 346 & 56.4 \\
& 2 & 313 & 51.0 \\
Place in birth order & 3 & 237 & 38.6 \\
& 4 & 46 & 7.5 \\
Child obesity index groups & Normal & 4 & 0.7 \\
& Overweight & 1 & 0.2 \\
& Mother & 27 & 4.4 \\
& Father & 515 & 83.9 \\
& Full-time & 5 & 4.6 \\
& Part-time & 30 & 4.9 \\
& Family business & 14 & 2.3 \\
& Unemployed & 550 & 89.6 \\
& Other & 12 & 2.0 \\
\hline
\end{tabular}




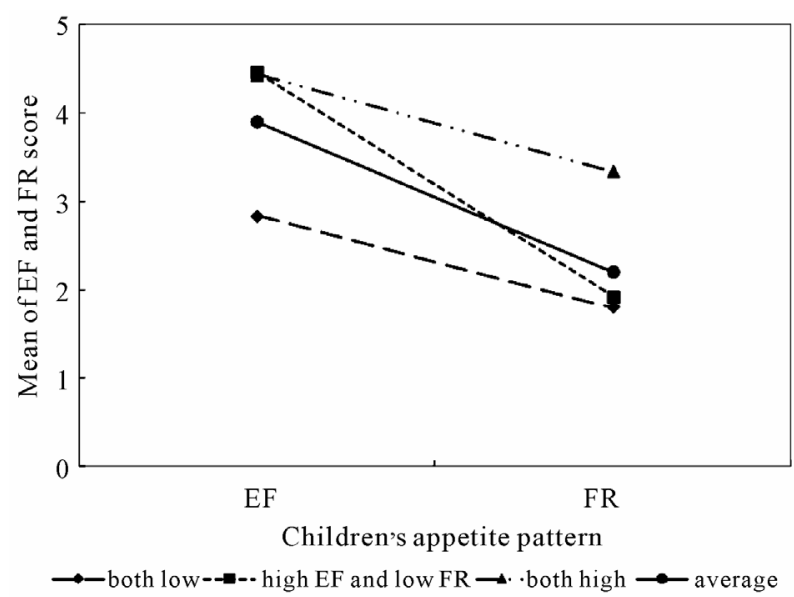

Figure 1. Appetite patterns classified by cluster analysis based on Enjoyment of Food (EF) and Food Responsiveness (FR) scores.

scores were $-0.34(-8.10-2.14) \%$ for "both low," -0.59 $(-5.69-5.47) \%$ for "high EF and low FR," and 2.52 $(-4.09-8.76) \%$ for "both high".

\subsection{Association between Maternal Feeding Practices and Children's Appetite Patterns}

Table 2 shows that children's appetite patterns were associated with parental feeding practices, particularly snacking behaviour. Mothers with "both low" children were more likely to use pressure to encourage children to eat than were mothers of children in the other groups. Mothers with children in the "both high" group were more likely to employ instrumental feeding than were mothers with "high EF and low FR" children. Emotional feeding was not significantly related to children's appetite patterns.

Mothers with "high EF and low FR" children were more likely to feed snacks once per day; on the other hand, mothers with "both high" children were more likely to feed snacks more than twice per day. Mothers with "both low" children were less likely to have an established snack time.

\section{Discussion}

The present study classified children's appetites into three patterns, measured the children's eating behaviours based on these patterns using cluster analysis, and examined associations between children's appetite patterns and parental feeding practices. The children's appetite patterns were classified as "both low", "high EF and low FR", and "both high" by cluster analysis. Our results showed that mothers with children in the "high EF and low FR" group did not use high pressure to eat, used instrumental feeding, and fed snacks appropriately.

\subsection{Children's Appetite Patterns and Body Types}

We compared children's obesity indices according to children's appetite patterns; our results were similar to those of previous studies in showing a positive association between appetite and body type [6-8]. Children with high FR had a higher mean obesity index than did children in the other groups, whereas children with low FR and high EF had a lower mean obesity index compared with the others.

This negative association between child adiposity and

Table 2. Association between maternal feeding practices and child's appetite pattern.

\begin{tabular}{|c|c|c|c|c|c|}
\hline & Both low & High EF and low FR & Both high & $\mathrm{Z} / \chi^{2}$ & $p$ \\
\hline \multicolumn{6}{|c|}{$\begin{array}{l}\text { Maternal feeding } \\
\text { (Median }(25 \text { th }-75 \text { th percentile) })\end{array}$} \\
\hline Instrumental feeding & $2.0(1.5-2.5)$ & $2.0(1.5-2.5)^{\mathrm{c}}$ & $2.3(1.7-2.8)^{\mathrm{d}}$ & 10.5 & 0.005 \\
\hline Emotional feeding & $1.4(1.0-2.0)$ & $1.4(1.0-1.9)$ & $1.4(1.0-1.8)$ & 2.1 & 0.349 \\
\hline \multicolumn{6}{|l|}{ Frequency of snack ${ }^{\mathrm{b}}(\mathrm{n}(\%))$} \\
\hline Once in a while or less & $27(14.0)$ & $28(9.8)$ & $10(8.5)$ & 16.2 & 0.003 \\
\hline Once per day & $111(57.5)$ & $193(67.7)$ & $60(51.3)$ & & \\
\hline Two or more times per day & $55(28.5)$ & $64(22.5)$ & $47(40.2)$ & & \\
\hline Established & $116(60.1)$ & $209(73.1)$ & $81(69.8)$ & 9.1 & 0.010 \\
\hline Not established & $77(39.9)$ & $77(26.9)$ & $35(30.2)$ & & \\
\hline
\end{tabular}

${ }^{\mathrm{a}}$ Kruskal-Wallis test; ${ }^{\mathrm{b}} \chi^{2}$ test; ${ }^{\mathrm{c}, \mathrm{d}}$ Values with different superscripts are significantly different $(p<0.05)$. 
parental pressure to eat has been consistently reported $[14,19]$. On the other hand, Webber et al. (2010) reported that the association between children's appetites and maternal feeding practices was maintained after controlling for children's body mass indices [10]. Thus, the child's weight and eating behaviour appear to be independently associated with maternal feeding practices. Mothers' feeding practices are responsive to their children's predispositions toward food.

\subsection{Children's Appetite Patterns and Maternal Feeding Practices}

It is possible that children who experience more pressure to eat may always have been difficult to feed, thus eliciting more pressure from their parents; in this case, low intake may cause parental pressure. Alternatively, it is also possible that children who are more pressured may learn to ignore or oppose parents' requests that they eat; in this case, low intake may be a consequence of parental pressure [19]. Two studies indicating that the use of pressure can foster negative affective responses to foods and thereby decrease liking of individual foods support the latter interpretation $[19,20]$. Additionally, evidence suggests that pressuring children to eat may diminish their ability to self-regulate their intake $[21,22]$. However, a bi-directional relationship is likely [23-25].

We showed that mothers with "high EF and low FR" children tended to establish snack times and to feed snacks once per day. The establishment of snack times is an important contributor to the creation of a healthy appetite because irregular and frequent snacking de- creases children's appetites at mealtimes. Furthermore, the present study showed that mothers with "both high" children used instrumental feeding practices with their children. Mothers may have controlled children's use of food because the children were more responsive toward food than were other children. Some previous studies have suggested that children whose food is controlled may have a stronger preference for the food [26-29]. Brown and Ogden (2004) discussed the possible outcome when parents encouraged their children to eat one food (the access food, e.g., vegetables) by promising another (the reward food, e.g., dessert), saying something like, "If you eat your vegetables, you can have your pudding" [30]. They suggested that this practice may increase children's preference for the reward food (pudding) even further, as pairing the two foods results in the reward food's being seen as more positive than the access food.

\subsection{Importance of Examination by Appetite Pattern}

The results of this study showed that "high EF and low FR" appetites were a result of appropriate feeding prac- tices. Previous studies have suggested that the high EF appetite is problematic because it fosters a propensity toward overweight status and obesity. However, the present study indicated that the effect of EF in the "high EF and low FR" pattern was similar to that in the "both high" pattern. Our findings suggest that "high EF and low FR" is not necessarily problematic. Thus, it is important to assess an appetite pattern using a combination of EF and FR. Furthermore, it is necessary to examine associations among appetite patterns.

\subsection{Limitations}

The present study had several limitations that should be acknowledged. First, this study was limited by its reliance on parental self-reports. The second limitation is that our sample was restricted to parents of children attending kindergarten in Tokyo, which limits the ability to generalise our findings. An additional limitation of the present study concerns its inability to demonstrate causal relationships; the data obtained via questionnaires allowed conclusions only about relationships between children's appetites and parental feeding practices and did not address the question of whether decreased appetites among children increased parental pressure to eat or if the reverse were the case. Future longitudinal research is needed to examine this question.

\subsection{Strengths}

Despite these limitations, this is the first study that classified children's appetite patterns by EF and FR based on scales from the CEBQ, which have previously been validated against behavioural measures of children's eating behaviour [9]. It was also the first to examine the association between children's appetite patterns and maternal feeding practices. The results of this study were consistent with a child-responsive model in which a child's appetite pattern influences maternal feeding practices, although the possibility of bidirectional effects cannot be ruled out. Further study is needed to understand the relationship between children's appetite patterns and maternal feeding practices.

\section{Conclusions}

This exploratory study suggested that the evaluation of children's appetite patterns using a combination of the $\mathrm{EF}$ and FR scales from the CEBQ may provide a more accurate understanding of children's eating behaviour. Additionally, children's appetite patterns were associated with maternal feeding practices. Because the present study was cross-sectional in design, longitudinal research is needed to understand the causal direction of the relationships between children's appetites and parental feeding practices (i.e., whether decreased appetites among 
children increased parental pressure to eat or the reverse). Practitioners may find it useful to take an interactional perspective in which children both influence and are influenced by maternal feeding practices. This approach may help practitioners to develop targeted interventions and provide better maternal guidance on managing eating behaviours in young children.

\section{REFERENCES}

[1] Y. Wang and T. Lobstein, "Worldwide Trends in Childhood Overweight and Obesity," International Journal of Pediatric Obesity, Vol. 1, No. 1, 2006, pp. 11-25. doi:10.1080/17477160600586747

[2] Japan Ministry of Health, Labour and Welfare, "The 21 Century Children Born Longitudinal Study," 2003. http://www.mhlw.go.jp/toukei/saikin/hw/syus-seiji/03/ke kka6.html

[3] A. Jansen, N. Theunissen, K. Slechten, C. Nederkoorn, B. Boon, S. Mulkens and A. Roefs, "Overweight Children Overeat after Exposure to Food Cues," Eating Behaviours, Vol. 4, No. 2, 2003, pp. 197-209. doi:10.1016/S1471-0153(03)00011-4

[4] S. Carnell and J. Wardle, "Appetite and Adiposity in Children: Evidence for a Behavirol Susceptibility Theory of Obesity," The American Journal of Clinical Nutrition, Vol. 88, No. 1, 2008, pp. 22-29.

[5] J. Wardle, C. A. Guthrie, S. Sanderson and L. Rapoport, "Development of the Children's Eating Behavior Questionnaire," Journal of Child Psychology and Psychiatry, Vol. 42, No. 7, 2001, pp. 963-970. doi:10.1111/1469-7610.00792

[6] E. F. C. Sleddens, S. P. J. Kremers and C. Thijs, "The Children's Eating Behaviour Questionnaire: Factorial Validity and Association with Body Mass Index in Dutch Children Aged 6-7," International Journal of Behavioral Nutrition and Physical Activity, Vol. 5, No. 1, 2008. doi:10.1186/1479-5868-5-49

[7] V. Viana, S. Sinde, J. C. Saxton, "Children's Eating Behaviour Questionnaire: Associations with BMI in Portuguese Children," The British Journal of Nutrition, Vol. 100, No. 2, 2008, pp. 445-450. doi:10.1017/S0007114508894391

[8] L. Webber, C. Hill, J. Saxton, C. H. M. V. Jaarsveld and J. Wardle, "Eating Behaviour and Weight in Children," International Journal of Obesity, Vol. 33, No. 1, 2009, pp. 21-28. doi:10.1038/ijo.2008.219

[9] S. Carnell and J. Wardle, "Measuring Behavioural Susceptibility to Obesity: Validation of the Child Eating Behavior Questionnaire," Appetite, Vol. 48, No.1, 2007, pp. 104-113. doi:10.1016/j.appet.2006.07.075

[10] L. Webber, L. Cooke, C. Hill and J. Wardle, “Associations between Children's Appetitetive Traits and Maternal Feeding Practices," Journal of American Dietetic Association, Vol. 110, No. 11, 2010, pp. 1718-1722. doi:10.1016/j.jada.2010.08.007

[11] J. O. Fisher, D. C. Mitchell, H. Smiciklas-Wright and L.
L. Birch, "Parental Influences on young Girls' Fruit and Vegetable, Micronutrient, and Fat Intake," International Journal of Obesity, Vol. 102, No. 1, 2002, pp. 58-64.

[12] C. V. Farrow, A. T. Galloway and K. Fraser, "Sibling Eating Behaviours and Differential Child Feeding Practices Reported by Parents," Appetite, Vol. 52, No. 2, 2009, pp. 307-302. doi:10.1016/j.appet.2008.10.009

[13] The Japanese Society of Child Health, "Preschool Children's Health Survey," 2000. http://jschild.or.jp/book/report_2000.html

[14] J. Blissett, C. Meyer and E. Haycraft, "Maternal and Paternal Controlling Feeding Practices with Male and Female Children," Appetite, Vol. 47, No. 2, 2006, pp. 212-219. doi:10.1016/j.appet.2006.04.002

[15] Mother and Children's Health and Welfare Association, "Survey on the Growth of Infants and Preschool Children, 2000," Boshi-Hoken Jigyoudan, Tokyo, 2002.

[16] The Japan Society for the Study of Obesity, "The Manual of Children's Obesity," Ishiyaku Publishers, Tokyo, 2004.

[17] L. L. Birch, J. O. Fisher, K. Grimm-Thomas, C. N. Markey, R. Sawyer and S. L. Johnson, "Confirmatory Factor Analysis of the Child Feeding Questionnaire: A Measure of Parental Attitudes, Beliefs, and Practices about Child Feeding and Obesity Proneness," Appetite, Vol. 36, No. 3, 2001, pp. 201-210. doi:10.1006/appe.2001.0398

[18] J. Wardle, S. Sanderson, C. A. Guthrie, L. Rapoport and R. Plomin, "Parental Feeding Style and the Intergenerational Transmission of Obesity Risk," Obesity Research, Vol. 10, No. 6, 2002, pp. 453-462. doi: $10.1038 /$ oby. 2002.63

[19] A. T. Galloway, L. M. Fiorito, L. A. Francis and L. L. Birch, “'Finish Your Soup!': Counterproductive Effects of Pressuring Children to Eat on Intake and Affect," $\mathrm{Ap}$ petite, Vol. 46, No. 3, 2006, pp. 318-323. doi:10.1016/j.appet.2006.01.019

[20] W. R. Batsell, A. S. Brown, M. E. Ansfield and G. Y. Pschall, "You Will Eat All of That!': A Retrospective Analysis of Forced Consumption Episodes," Appetite, Vol. 38, No. 3, 2002, pp. 211-219. doi:10.1006/appe.2001.0482

[21] L. L. Birch, L. McPhee, B. C. Shoba, L. Steinberg and R. Krehbiel, "Clean up Your Plate. Effects of Child Feeding Practices on the Condition of Meal Size," Learning and Motivation, Vol. 18, No. 3, 1987, pp. 301-317. doi:10.1016/0023-9690(87)90017-8

[22] J. L. Carper, F. J. Orlet and L. L. Birch, "Young Girls' Emerging Dietary Restraint and Disinhibition Are Related to Parental Control in Child Feeding," Appetite, Vol. 35, No. 2, 2000, pp. 121-129. doi:10.1006/appe.2000.0343

[23] M. S. Faith and J. Kerns, "Infatnt and Child Feeding Practices and Childhood Overweight. The Role of Restriction," Maternal and Child Nutrition, Vol. 1, No. 3, 2005, pp. 164-168. doi:10.1111/j.1740-8709.2005.00024.x

[24] A. T. Galloway, L. Fiorito, Y. Lee and L. L. Birch, "Pa- 
rental Pressure, Dietary Patterns, and Weight Status among Girls Who Are 'Picky Eaters'," Journal of the American Dietetic Association, Vol. 102, No. 4, 2005, pp. 58-64.

[25] A. K. Ventura and L. L. Birch, "Does Parenting Affect Children's Eating and Weight Status?" International Journal of Behavioural Nutrition and Physical Activity, Vol. 5, No. 15, 2008. doi:10.1186/1479-5868-5-15

[26] L. L. Birch, D. Birch, D. Marlin and L. Kramer, "Effects of Instrumental Eating on Children's Food Preferences," Appetite, Vol. 3, No. 2, 1982, pp. 125-134.

[27] M. Lepper, G. Sagotsky, J. L. Dafoe and D. Greene, "Consequences of Superfluous Social Constraints: Effects on Young Children's Social Influences and Subsequent Intrinsic Interest," Journal of Personality and Social
Psychology, Vol. 42, No. 1, 1982, pp. 51-65. doi:10.1037/0022-3514.42.1.51

[28] L. L.Birch, D. Marlin and J. Rotter, "Eating as the 'Means' Activity in a Contingency: Effects on Young Children's Food Preference," Child Development, Vol. 55, No. 2, 1984, pp. 431-439. doi:10.2307/1129954

[29] J. Newman and A. Taylor, "Effects of a Means-End Contingency on Young Children's Food Preferences," Journal of Experimental Psychology, Vol. 53, No. 2, 1992, pp. 200-216.

[30] R. Brown and J. Ogden, "Children's Eating Attitudes and Behavior: A Study of the Modeling and Control Theories of Parental Influence," Health Education Research, Vol. 19, No. 3, 2004, pp. 261-271. doi:10.1093/her/cyg040 